\title{
An Applied Research on Improved Watershed Algorithm in Medical Image Segmentation
}

\author{
BenZhai Hai ${ }^{1,2}$, RuiYun Xie ${ }^{3}$ and PeiYan Yuan ${ }^{1}$ \\ ${ }^{1}$ College of Computer \& Information Engineering, Henan Normal University, \\ Xinxiang, Henan, 453007, China \\ ${ }^{2}$ Information Engineering college, Wuhan University Of Technology, \\ Wuhan ,Hubei ,430070, China \\ ${ }^{3}$ Department of Computer Science and Technology, Henan Institute of \\ Technology, Xinxiang, Henan, 453000, China \\ 271932557@qq.com
}

\begin{abstract}
The image segmentation technology is of great significance to the target identification. The watershed segmentation algorithm has wide application in image segmentation. The traditional watershed segmentation often causes the problems of over segmentation and noise sensitivity. Therefore, a medical image segmentation algorithm is proposed based on K-means clustering algorithm and improved watershed algorithm. First, $K$ - means clustering algorithm is used for initial segmentation, and then the concept of similarity is put forward to improve the original watershed algorithm. Finally, the adjacent tiles of the initial segmentation is merged. The magnetic resonance image is regarded as the segmentation object. The experimental result shows that the proposed algorithm effectively solves the problem of the over-segmentation of traditional watershed algorithm, and achieves a satisfactory effect for the image segmentation.
\end{abstract}

Keywords: K-means;improved;watershed; image segmentation

\section{Introduction}

In the analysis of the computer vision and image, how to segment effectively the target object from the image partition has been one of classic problems, which determines the image quality of the final analysis and the result of the pattern recognition. Image segmentation means that the different areas with special meanings are segmented, which makes these areas not intersect each other, and each area should satisfy the consistency condition of the particular area. Once the image is segmented, it can be further processed, such as the image retrieval classification and recognition based on the content. As a result, the image segmentation is a very important research filed in the image processing and the pattern recognition.

The image segmentation is a process in which an image is divided into several parts or subsets according to certain rules, which is a key step from image processing to image analysis [1]. Watershed transform has been widely used in the image segmentation and the processing. The literature [2] uses watershed transform to segment the target of the gray and white matter from magnetic resonance(MR) images. The algorithm introduces the concept of ridge and catchment basin in geography into image processing. For example, the pixel gray value $\mathrm{I}(\mathrm{x}, \mathrm{y})$ is considered as the altitude at the beginning of a low but correct segmentation threshold. With the rise of threshold, the best segmentation effect can be achieved, so that the adjacent targets will not be merged to solve those problems that the close target cannot use global threshold segmentation properly. Watershed algorithm has the rapid, simple and direct advantages. It has better segmentation effect for 
the quality of low contrast image, but its subsequent processing requirement is not high. However, this method is sensitive to noise with the over segmentation problem [3].

In the terms of the image segmentation, fuzzy clustering segmentation algorithm [4] attracts more attention. The fuzzy clustering segmentation uses the membership degree to present a pixel belonging to the category. The literature [5] uses fuzzy $C$-means algorithm for MR image segmentation, but the algorithm increases the computational complexity. The anti-noise performance of this algorithm needs to be further improved. Compared with the fuzzy $C$-means, $K$-means algorithm has the advantages of low computational complexity, and the overlapping degree of segmentation targets is low [6].

An algorithm of image segmentation is proposed based on $K$-means clustering algorithm and improved watershed algorithm. First, $K$-means clustering algorithm is used for initial segmentation, and then the concept of similarity is put forward to improve the original watershed algorithm. Finally, the adjacent tiles of the initial segmentation is merged. The experimental result shows that the proposed algorithm effectively solves the problem of the over-segmentation of traditional watershed algorithm, which has reference value for image segmentation and recognition

\section{2. $K$-Means Clustering Algorithm}

The $K$-means clustering algorithm is a kind of clustering algorithm of the emblem, which can be used to initializing the split. From past experiences, we can observe that there are many similar gray areas in medical image, which will cause a lot of local minimum values. So we can use clustering algorithm for the initial clustering, to reduce the over-segmentation phenomenon of watershed algorithm. At the same time, the $K$ means clustering algorithm is a rapid, simple and effective classification algorithm. This study takes the bones in the medical image as experiment objects, which is more suitable for the application of $K$-means algorithm [7].

$(x, y)$ is set the coordinate of the digital image. $f(x, y)$ is treated as its gray scale function. $\mathrm{Q}_{\mathrm{j}}{ }^{\mathrm{i})}$ presents $j$ region after clustering for $i$ time. $\mu_{j}{ }^{(i+1)}$ stands for $j$ region after clustering for $i+1$ time. $\mu_{j}^{(i+1)}$ means after clustering for $i+1$ time the average value (clustering center) of $j$ classification.

$K$-means clustering algorithm is used for initial classification process, which is shown as follows:

(1) As for initialization clustering center $m_{1}{ }^{(1)}, m_{2}{ }^{(1)}, \ldots, m_{K}{ }^{(1)}$, the histogram is used to choose $K$ grey value as the initial average value.

(2) As for each point $(x, y)$, according to the following criteria, the nearest cluster center is found, and assigned to this class.

$\left|f(x, y)-n_{l}^{(j)}\right|<\left|f(x, y)-n_{j}^{(j)}\right|$

After update is complete, a new clustering $\mathrm{Q}_{j}^{(i+1)}$ is received.

(3) Each kind of clustering center $\mu_{j}^{(i+1)}$ is updated.

$X^{2}=$ 檍 $_{j=1(m, n) \hat{1}}^{Q_{j}^{(i)}}\left|f\left(x, y-n f^{(j+1)}\right)\right|^{2}$

(4) The criterion function $\xi^{2}$ is calculated. If $\xi^{2}$ is for convergence, the clustering process is over, otherwise, it changes

$n \dot{h}^{+1}=\frac{1}{N_{j}} \underset{(x, y) \mathfrak{\complement} Q_{j}^{(i)}}{\stackrel{\circ}{\mathrm{a}}} f(x, y)$ 
Figure 2, shows the initial segmentation effect after clustering by $K$-means clustering algorithm.

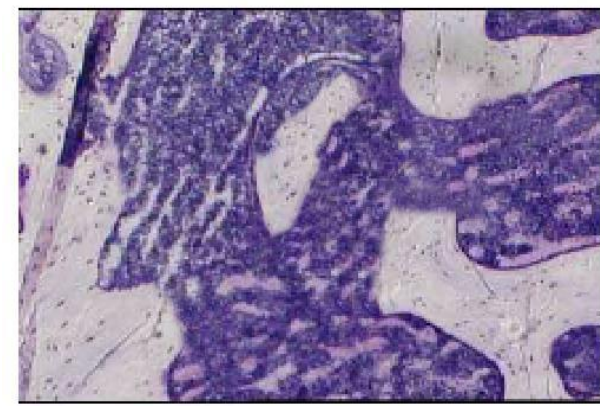

Figure 1. Bone Medical Images

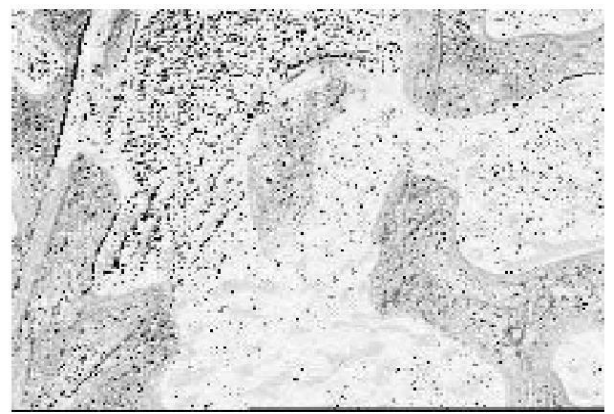

Figure 2. Bone Medical Images after Clustering by $K$-Means

\section{Improved Watershed Segmentation Algorithm}

Watershed image segmentation algorithm is widely used, but its disadvantage is oversegmentation. That is to say, the image is divided into too many small areas, which makes the interested targets covered in it. Watershed transform is a typical segmentation method based on region. The concept of this method is introduced from geography. The watershed is a line to separate rainwater accumulation area (catchment basin). Another idea is that the catchment basin is being immersed in water, its local minimum values (the lowest point of catchment basin) has a hole. The lowest place of the catchment basin will be slowly put into. When in the water of different catchment basins is put together, a dam will be built to prevent water from gathering. When the immersion process is over, each basin is flooded, and completely surrounded by the dam. These dams is known as a watershed line or watershed in short [8].

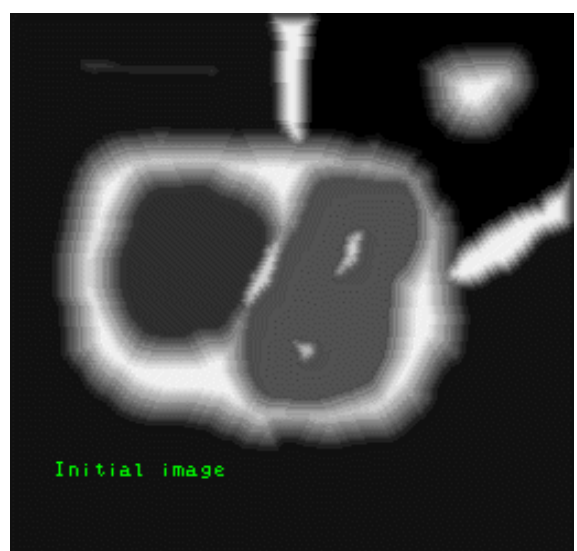

(a)The Initial State of the Watershed 


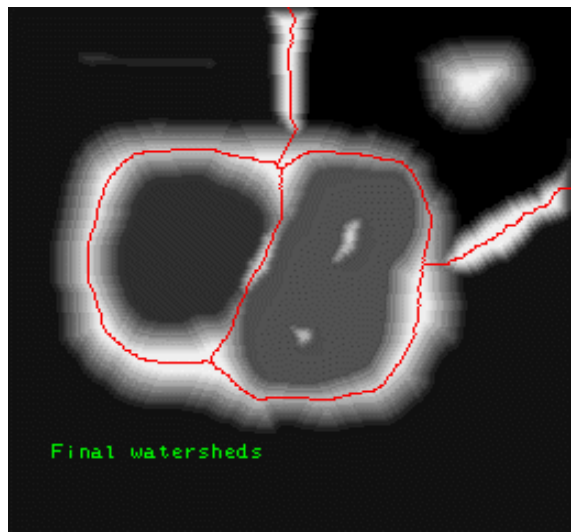

(b) The Image Segmentation of the Watershed

\section{Figure 3. The Algorithm Principle of the Watershed Segmentation}

The concrete steps of watershed segmentation algorithm are shown as follows [9]:

$M_{1}, \quad M_{2}, \ldots M_{k}$ is set as the local minimum point of the image $f(x, y), B_{1}, B_{2}, \ldots, B_{k}$ is the set of all points in the catchment basin which each local minimum point is corresponding to, $B^{n}{ }_{k}$ stands for all point sets in the $n$ period catchment basin $B_{k}$. $B^{n}$ stands for all $B^{n}{ }_{k}$ set. $f_{\min }$ and $f_{\max }$ stand for minimum and maximum of image gray levels respectively. Based on the above description, the formula is drawn.

$B^{n}=\bigcup_{k=1}^{K}\left(B_{k}^{n}\right)$

And the set of all catchment basins:

$B^{f_{\max }+1}=\bigcup_{k=1}^{K}\left(B_{k}\right)$

$T(n)$ stands for the point set of flooded point in the image, that is $T(n)=\{(\mathrm{i}, \mathrm{j}) \mid f(i, j)<n\}$, and tht following formulate is drawn

$B_{k}^{n}=B_{k} \cap T(n)$

Therefore, the binarization image can be constructed to be corresponding to the original image. If original image point $(i, j) \perp T(n)$, this point is set as 0 ,otherwise it is set as 1 . Through the " 0 " count, it is possible to know the number of points in the $n$ stage under water level. The specific method is as follows:

The initial collection of the catchment basin is set as $B^{f_{\min }+1}=T\left(f_{\min }+1\right)$, and then the algorithm enters into the recursive stage. The water level increases with the integer value from $\mathrm{n}=f_{\min }+1$ to $f_{\max }+1$, and records every stage flooded point set $T(n)$. If the $n$ period $T(n)$ has Q connected component $B_{q}^{n}$, where $q \leq Q$. It is assumed that in the $n$-1period, $B^{n-1}$ has been constructed. For each $q$ value, it is necessary to compare connected component $B^{n}{ }_{q}$ with $B^{n-1}$, and the two meet different relations and they will perform different operations. (1) $B_{q}^{n} \cap B^{n-1}=$ ? , a new connected component appears and a new catchment basin turns up. $B^{n}{ }_{q}$ and $B^{n-1}$ are combined.

(2) $B_{q}^{n} \cap B^{n-1}=$ ? includes a connected component of $B^{n-1}$, which shows that $B^{n-1}$ has its catchment basin. The corresponding parts of $B^{n}{ }_{q}$ and $B^{n-1}$ are combined. 
(3) $B_{q}^{n} \cap B^{n-1}=$ ? includes two or more connected components of $B^{n-1}$. It is necessary to build the watershed in $B^{n}$. The watershed construction adopts morphological expansion operation to prevent the combination of the catchment basin.

The watershed algorithm above is used for medical image segmentation, which causes often over-segmentation phenomenon. This study proposes an improved watershed algorithm, which is mainly used for the subsequent processing to the over segmentation to combine over-segmentation parts, according to certain rules. Specific steps are shown as follows:

(1)After the initial segmentation, the image is segmented as $N$ pieces. Each piece is set as $S_{i}$, and the size of each piece is set as $n_{i}, 0<i<N$. The grayscale average $M_{i}$ of each piece is calculated

$M_{i}=\frac{1}{n_{i}} \underset{(x, y) \hat{1} R_{i}}{\stackrel{\circ}{I}} I(x, y)$

(2) $S_{i}$ and $S_{j}$ are set as two pieces, and then the following measures are defined.

The grayscale average difference between two adjacent pieces is shown as:

$M_{i j}=\sqrt{\left(M_{i}-M_{j}\right)^{2}}$

(3) $S_{i}$ and $S_{j}$ are set as two adjacent segmentation pieces, and $\left(x_{i}, y_{i}\right) \in S_{i}$ and $\left(x_{j}, y_{j}\right) \in S_{j}$ are the points of the common boundary of $S_{i}$ and $S_{j}$; and $\left(x_{i}, y_{i}\right)$ and $\left(x_{j}, y_{j}\right)$ meet 4-domain relation . The number $N_{i j}$ is the point of the common boundary.

$P_{i j}=\frac{1}{N_{i j}} \underset{\left(x_{i}, y_{i}\right)\left(x_{j}, y_{j}\right)}{\stackrel{\circ}{\left(I\left(x_{i}, y_{i}\right)-I\left(x_{j}, y_{j}\right)^{2}\right.}}$

(4)The similarity between two segmentation pieces is defined

$C_{i j}=\frac{\sqrt{\left(M_{i j}+P_{i j}\right)^{2}}}{2}$

(5) Combining two adjacent pieces according to the threshold value

The adaptive threshold $T$ is generated based on the gradient magnitude image histogram in literature [9], and according to the following formula, adjacent segmentation pieces are handled.

if $C_{i j}>\mathrm{T} \quad S_{i}$ and $S_{j}$ are cømbined

if $C_{i j}<\mathrm{T} \quad S_{i}$ and $S_{j}$ are not combined

\section{The Results and Analysis of the Experiments}

The results of the proposed algorithm are compared with the results of the traditional watershed algorithm segmentation. The subjects include 100 images of 2D medical bone, and medical bone images is made up of bone and soft tissue, fat, and background, so in $K$ means algorithm, $\mathrm{K}=4$. Because the most main problem of the watershed algorithm is the over-segmentation phenomena, the comparative index is segmentation piece. $91 \%-96 \%$ of 90 bone medical image segmentation pieces is combined, and 85\%-90\% of 10 bone medical image segmentation pieces is merged. The proposed algorithm is compared with the traditional watershed algorithm segmentation pieces as shown in Figure 4. 


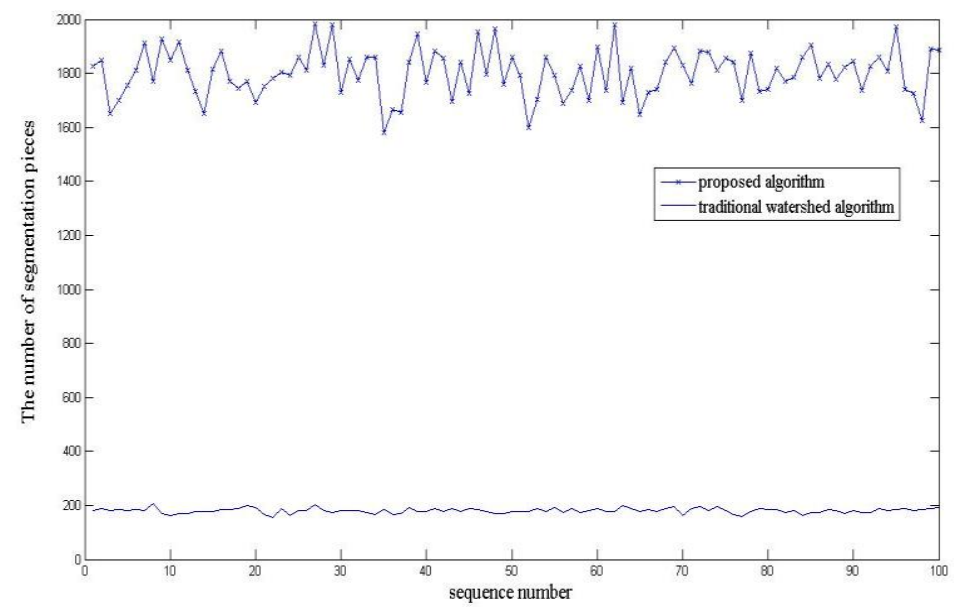

Figure 4. The Number of Segmentation Pieces Comparison between the Proposed Algorithm and the Traditional Watershed Algorithm

If K-means clustering algorithm is not used, but the improved watershed algorithm is directly used for human bone MR image segmentation. The result of image segmentation is 278 pieces. Therefore, the $K$-means clustering algorithm to a certain extent improves the over-segmentation of the watershed algorithm. Figure 5, is the original MR image of the bones. Based on the experimental results, in Figure 6, the original watershed algorithm for segmentation of MR images has serious over-segmentation phenomenon. In Figure 7, the improved watershed algorithm segments basically bone structure partition, and there is no segmentation, which have improved significantly compared to Figure 6.

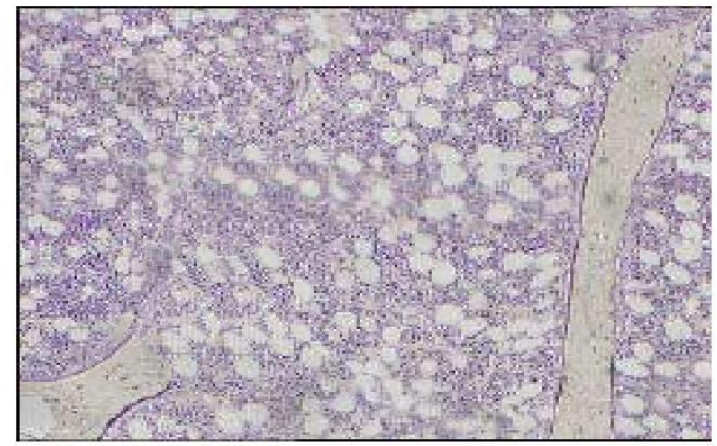

Figure 5. The Original Image of the Medical Bone

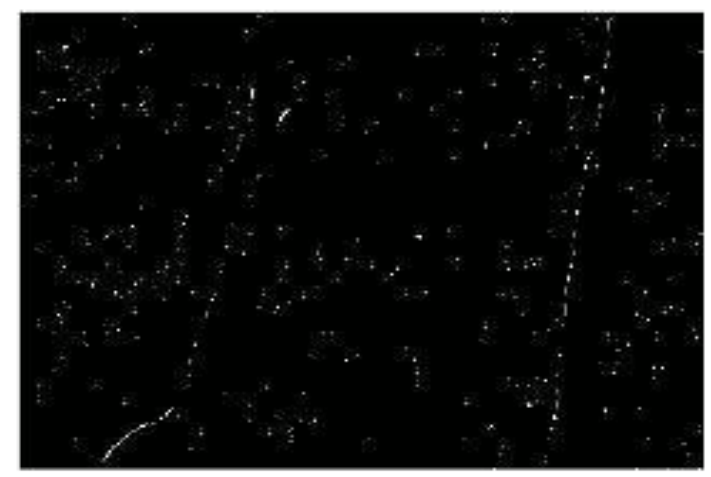

Figure 6. The Image Segmentation Based on the Original Watershed Algorithm 


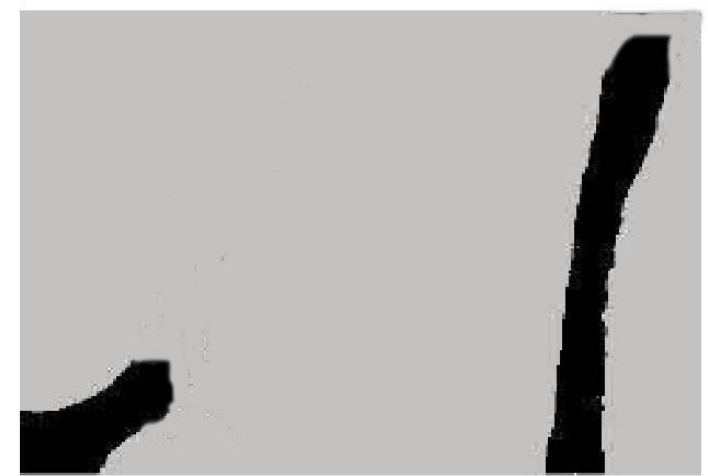

\section{Figure 7. The Image Segmentation of the Improved Watershed Segmentation Algorithm}

The proposed algorithm and the original watershed segmentation algorithm are applied to the image segmentation of the medical bone. The results show the proposed algorithm has better segmentation effect, which has important significance to the medical diagnosis.

\section{Conclusion}

An image segmentation algorithm is proposed based on K-means clustering algorithm and improved watershed algorithm. First, $K$-means clustering algorithm is used for initial segmentation, and then the concept of similarity is put forward to improve the original watershed algorithm. Finally, the adjacent tiles of the initial segmentation is merged. The magnetic resonance image is regarded as the segmentation object. The experimental result shows that the proposed algorithm effectively solves the problem of the oversegmentation of traditional watershed algorithm, and achieves a satisfactory effect for the image segmentation.

\section{Acknowledgments}

The study was supported by following Funds.

1. Youth Science Fund of Henan Normal University[No.2014QK30].

2. Science and technology project of Henan Province [No.162102310442]

3. National Natural Science Foundation of China [No.U1404602]

4. Key scientific research projects of University of Henan Province [No.15B520006].

\section{References}

[1] V. Grau, R. Kikinis, M. Alcaniz and S. K. Warfield, "Cortical gray matter segmentation using an improved watershed transform", Proceedings 25th Annual Int. Conf. of Engineering in Medicine and Biology Society, vol. 1, (2009), pp. 618-621.

[2] V. Grau, A. U. J. Mewes, M. Alcaniz, R. Kikinis and S. K. Warfield, "Improved watershed transform for medical image segmentation using prior information", IEEE Transactions on Medical Imaging, vol. 23, no. 4, (2010), pp. 447-458.

[3] X. H. Yuan and X. D. X. Liang, "The image segmentation based on the morphological filter and the goal of watershed", Journal of data acquisition and processing, vol. 18, no. 4, (2010), pp. 455- 489.

[4] J. B. T. M. Roerdink and A. Meijster, "The watershed transform: Definitions, algorithms and parallelization strategies”, Fundamental Informaticae, vol. 41, (2009), pp. 187-228.

[5] L. Li, L. Chunmei and J. Jinli, "Improved clustering algorithm in the application of medical image segmentation", Journal of mathematical medicine journal, vol. 6, (2010).

[6] L. Ren, H. C. Lai, Q. Chen and X. Wang, "A cotton segmentation algorithm based on improved k-means clustering and HSV model", vol. 5, (2013), pp. 22-24.

[7] S. Yu, Y. H. Zhou and R. Zhang, "Digital image processing", Shanghai, Shanghai Jiaotong university press, (2007).

[8] K. Dong and D. L. Jiang, "Ore image segmentation algorithm based on improved watershed transform", Computer engineering and set, vol. 3, (2013), pp. 100-103. 
[9] X. Li, J. Wu and G. F. Zhang, "Color image segmentation Based on watershed and region merging", Journal of electronic measurement and instrument, vol. 27, no. 3, (2013), pp. 23-25.

\section{Authors}

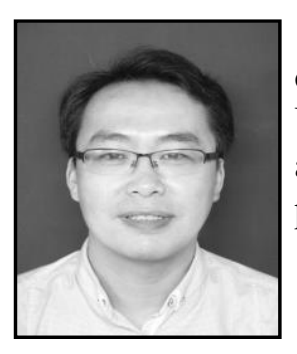

BenZhai Hai, received his Master Degree from Wuhan University of Technology in 2009. He is now an Lecturer of Henan Normal University in China. His research interests include computer application; Computer Network.He has published more than 20 papers in journals and conferences.

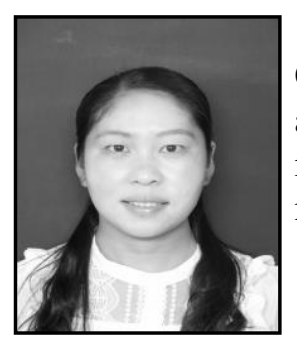

RuiYun Xie, received her Master Degree from Wuhan University Of Technology in 2009. She is now an Lecturer of Henan Mechanical and Electrical Engineering College in China. His research interests include computer application, Computer Network.He has published more than 10 papers in journals and conferences.

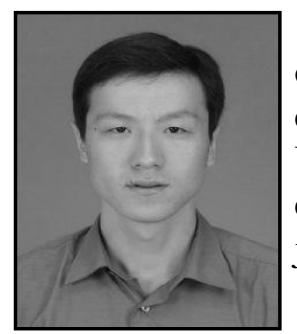

Peiyan Yuan, received his Doctor Degree from Beijing University of Posts and Telecommunications in 2014. He is now an professor of computer and Information Engineering College of Henan Normal University, master tutor. His research interests are the opportunity to calculate the D2D network. He has published more than 40 papers in journals and conferences. 